\title{
Invitro Assessment of Antibacterial and Antioxidant Property of Biofabricated Silver Nanoparticles using Aqueous Extract of Bauhinia Tomentosa
}

\author{
Mohanapriya. P \\ PG Student, Department of Nanoscience and Technology, \\ G.K.M College of Engineering and Technology, Perungalathur, Chennai, Tamil Nadu, India
}

\begin{abstract}
How to cite this paper: Mohanapriya. $P$ "Invitro Assessment of Antibacterial and Antioxidant Property of Biofabricated Silver Nanoparticles using Aqueous Extract of Bauhinia Tomentosa" Published in International Journal of Trend in Scientific Research and Development

(ijtsrd), ISSN: 24566470, Volume-3 | Issue-3, April 2019, pp.1127-1129, URL: https://www.ijtsrd.c om/papers/ijtsrd22 780.pdf

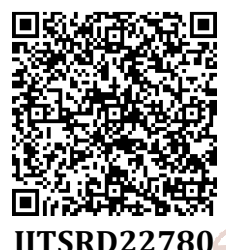

Copyright (C) 2019 by author(s) and International Journal of Trend in Scientific Research and Development Journal. This is an Open Access article distributed under the terms of the Creative Commons

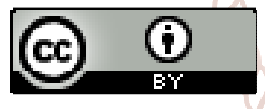
Attribution License (CC BY 4.0) (http://creativecommons.org/licenses/ by/4.0)

\section{INTRODUCTION}

Nanotechnology is the emerging technology in modern research field, which deals with design, manufacture and application of matter/ material by controlling its size and shape at atomic scale in $10^{-9} \mathrm{~m}(\mathrm{~nm})$. Metal nanoparticles have attracted for biomedical application due to its reduced size and large surface area to volume ratio utilized in wound healing, bio imaging, drug delivery and disease treatment [1]. Silver nanoparticles have broad range of importance in various fields like medicine, textile and cosmetics [2]. Green approach to synthesise metal nanoparticles using microbes and plant gained significance such as simple, low cost, non toxic, ecofriendly and one step process compared to other conventional methods [3]. Use of plant extract to synthesise metal nanoparticles is rapid than the microbe mediated synthesis. Because, since the microbes are biohazardous to human kind. The presence of various bioactive components in the plant extract act to reduce the size of the metal particles to nanosize [4]. Bauhinia tomentosa is a yellow bell orchid plant widely used in folklore medicine to treat various diseases like diarrohea, leprosy, wound ulcers, skin disorders, dysentery and diabetes [5]. It belongs to Fabacea family, commonly known as Kanjana in tamil. The plant as wide range of phytochemical compounds such as carbohydrates, proteins, alkaloids, flavonoids, glycosides, terpenoids and phenols which are for medicinal value of the plant [6]. Thus, in the present investigation the bio fabrication of silver nanoparticles was done using aqueous extract of Bauhinia tomentosa. The synthesised silver nanoparticles were characterized by using UV- Vis spectrophotometer and FTIR analysis. Further the silver nanoparticles were subjected to antibacterial activity by using well diffusion method and antioxidant property by hydrogen peroxide assay.

\section{Experimental}

\subsection{Collection Of Plant:}

The diseased free plant leaves was collected in the month of November from the garden of Kanchipuram district, Tamil Nadu and identified as Bauhinia tomentosa based on their morphological characters.

\subsection{Preparation Of Plant Extract:}

The collected plant leaves was cleaned with tap water to remove dust particles and followed by distilled water. $20 \mathrm{~g}$ of 
fresh leaves were weighed and with help of mortar and pestel the extract was prepared with using $50 \mathrm{~mL}$ of deionised water. And extract was filtered using Whatmann filter paper No:1. The collected filtrate was heat treated for about $80^{\circ} \mathrm{C}$ for 20 minutes. And the cured extract was used for the further study.

\subsection{Bio Fabrication Of Silver Nanoparticles:}

$1 \mathrm{mM}$ concentration of silver nitrate solution was prepared using deionised water. To the $20 \mathrm{~mL}$ of $1 \mathrm{mM}$ of silver nitrate solution, $20 \mathrm{~mL}$ of the aqueous plant extract was added under continuous stirring. The solution mixture was kept under dark condition for $1 \mathrm{hr}$. The color change from dark green into reddish brown indicates the formation silver nanoparticles.

\subsection{Purification Of Silver Nanoparticles:}

The synthesized silver nanoparticle solution was centrifuged at $8000 \mathrm{rpm}$ for 10 minutes. The supernatant was collected and stored for further settlement of nanoparticles. The pellet was dried at $50^{\circ} \mathrm{C}$ in hot plate and powder form of silver nanoparticles was collected.

\subsection{Characterization:}

\section{UV- Visible Spectrophotometer And FT-IR Analysis:}

The synthesized silver nanoparticle solution was analysed using UV-Visible spectrophotometer. The peak obtained at the range of 400 to $500 \mathrm{~nm}$ due to surface Plasmon resonance which confirms the formation silver nanoparticles. The powdered silver nanoparticle was mixed with $\mathrm{KBr}$ to make the pellet and analysed to know the presence of functional groups using FT-IR.

\subsection{Antibacterial Activity:}

The antibacterial activity for synthesized silver nanoparticles was studied using agar well diffusion method against E. coli, S. aureus and S. typhi with four different concentrations $30 \mu \mathrm{L}, 60 \mu \mathrm{L}, 90 \mu \mathrm{L}$ and $120 \mu \mathrm{L}$. Muller Hinton agar medium was prepared and poured into the sterile petriplate and allowed to get solidifies. The wells were punched using sterile cork borer and inoculated with one day over night bacterial cultures and wells were loaded with synthesised silver nanoparticles solution of desired concentrations respectively.

\subsection{Antioxidant Property:}

The antioxidant property of synthesized silver nanoparticles was studied using hydrogen peroxide scavenging assay. The modified Dehpour method was used to determine the scavenging action of the synthesized silver nanoparticles towards Hydrogen peroxide using standard ascorbic acid. The solution of hydrogen peroxide $(40 \mathrm{mM})$ was prepared in phosphate buffer $(\mathrm{pH}=7.4)$. The silver nanoparticles of different concentration were taken in test tubes. The hydrogen peroxide prepared with phosphate buffer solution was added to it and incubate for 10 minutes. The absorbance was measured at $560 \mathrm{~nm}$ using UV spectrophotometer against blank solution containing phosphate buffer without hydrogen peroxide. The percentage of hydrogen peroxide scavenging activity of the silver nano materials was calculated by using the formula:

\section{$\%$ Scavenging \\ $=\underline{\text { Absorbance of Control }- \text { Absorbance of Sample X } 100}$} Absorbance of Control

\section{Result and Discussion:}

\subsection{Bio fabrication of Silver Nanoparticles:}

The aqueous extract of Bauhinia tomentosa is mediated to reduce the size silver ions into silver nanoparticles. It was confirmed by the visual observation of solution mixture color change into reddish brown (FIGURE 3.1). The silver nanoparticles were purified by centrifugation at $8000 \mathrm{rpm}$ for 10 minutes (FIGURE 3.2).

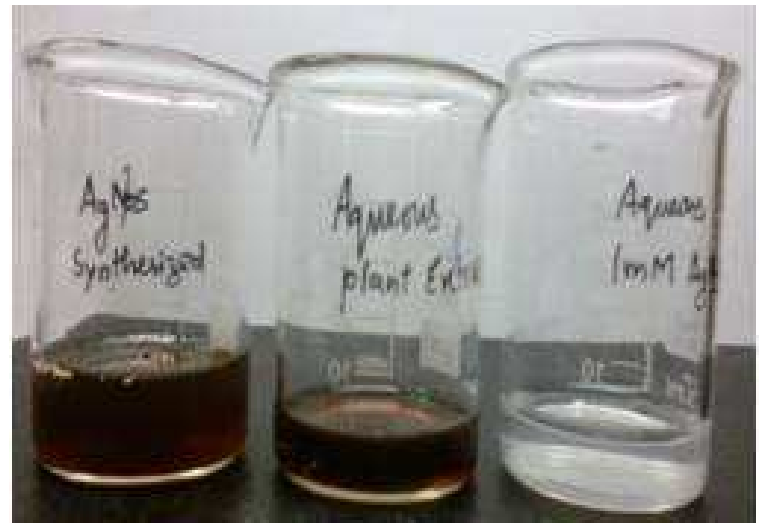

FIGURE 3.1- Synthesizing of Silver Nanoparticles Using Aqueous Extract of Bauhinia tomentosa

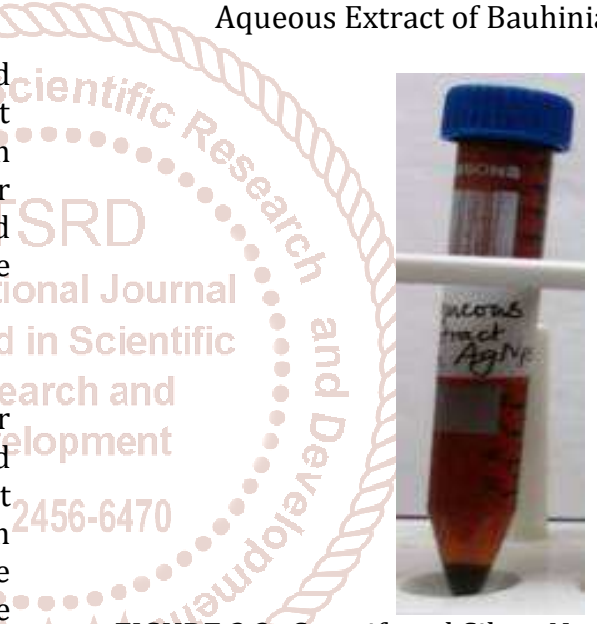

FIGURE 3.2-Centrifuged Silver Nanoparticles Solution

\subsection{Characterization:}

\subsubsection{UV- Visible Spectrophotometer Analysis:}

The synthesized silver nanoparticles were characterized using UV-Vis spectrophotometer for primary conformation to know the formation of silver nanoparticles. The UV- Vis spectra showed the absorbance peak obtained at $440.0 \mathrm{~nm}$ (FIGURE 3.3). It is due to the Surface Plasmon Resonance of the surface electrons of silver nanoparticles. The appearance of single indicates that the sphere shape of silver nanoparticles.

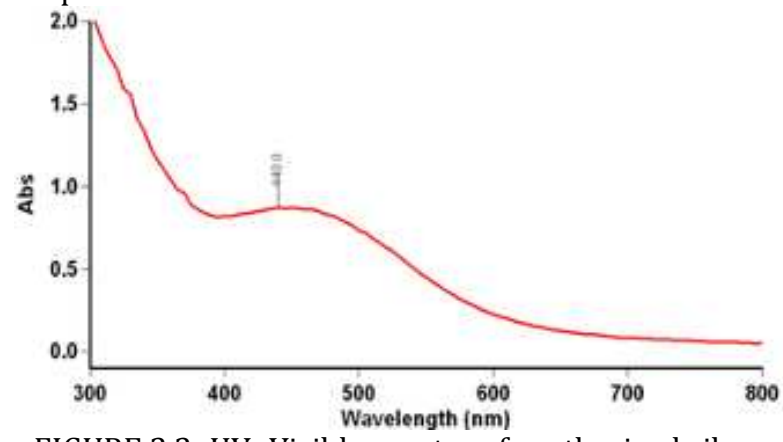

FIGURE 3.3- UV- Visible spectra of synthesized silver nanoparticles using aqueous extract of Bauhinia tomentosa 


\subsubsection{FT-IR Analysis:}

To analyze the presence of functional groups in the synthesized silver nanoparticles were performed. The FT-IR spectra registered the presence of phenol group at 3263.56 $\mathrm{cm}^{-1}, \mathrm{C}-\mathrm{H}$ stretch at $2916.37 \mathrm{~cm}^{-1}, \mathrm{C}=\mathrm{C}$ bending at 1602.85 $\mathrm{cm}^{-1}, \mathrm{C}-\mathrm{O}$ stretch at $1020.34 \mathrm{~cm}^{-1}, \mathrm{C}-\mathrm{H}$ bonding at 1346.31 $\mathrm{cm}^{-1}$ and Aromatic C-H bending at $821.68 \mathrm{~cm}^{-1}$. (FIGURE 3.4)

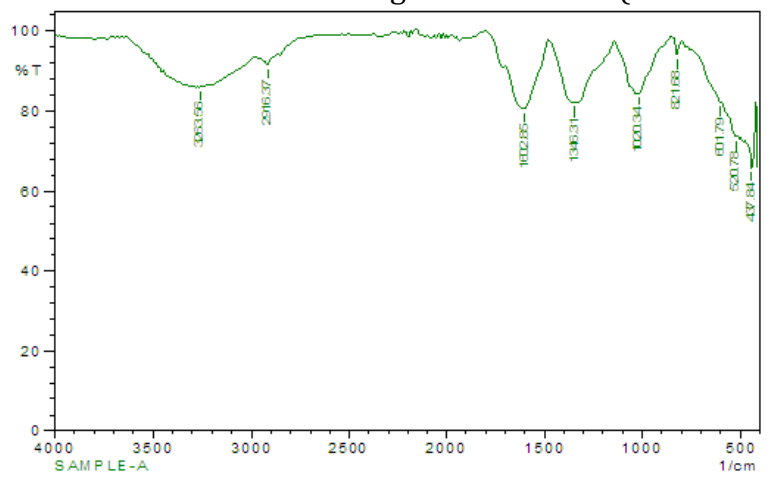

FIGURE 3.4- FTIR spectra of synthesized silver nanoparticles using aqueous extract of Bauhinia tomentosa

\subsection{Antibacterial Activity:}

The synthesized silver nanoparticles were subjected to antibacterial activity by well diffusion method. The bacterial cultures such as Escherichia coli, Salmonella typhi and Staphylococcus aureus were used for the bactericidal activity study. The minimum zones of inhibition of the plates (FIGURE 3.5) were measured in milimeter measurement (Table 3.1). The test was performed for duplicate plates.

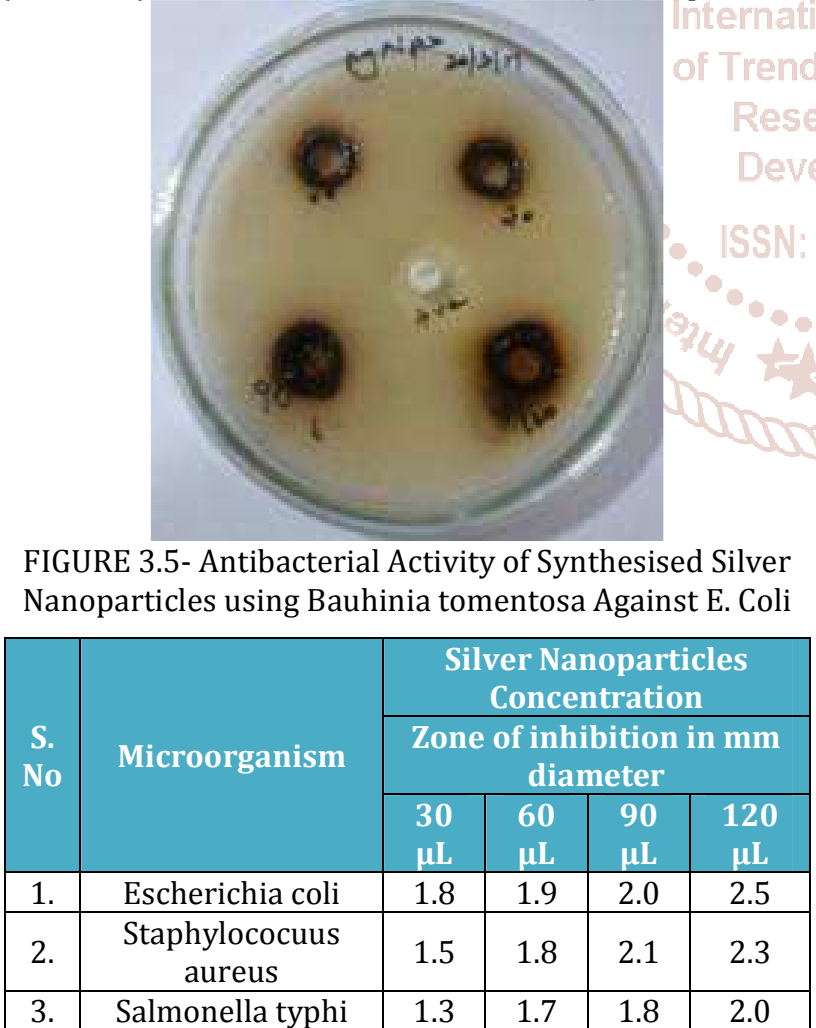
Internationa

TABLE 3.1- Antibacterial Activity Of Synthesized Silver Nanoparticles - Zone Of Inhibition

\subsection{Antioxidant Property:}

The synthesized silver nanoparticles were subjected to hydrogen peroxide scavenging assay to analyse the antioxidant property. The silver nanoparticles showed gradual increase in antioxidant property according to their concentration utilized for the assay, ascorbic acid were used as standard. (FIGURE 3.6)

Hydrogen Peroxide Assay

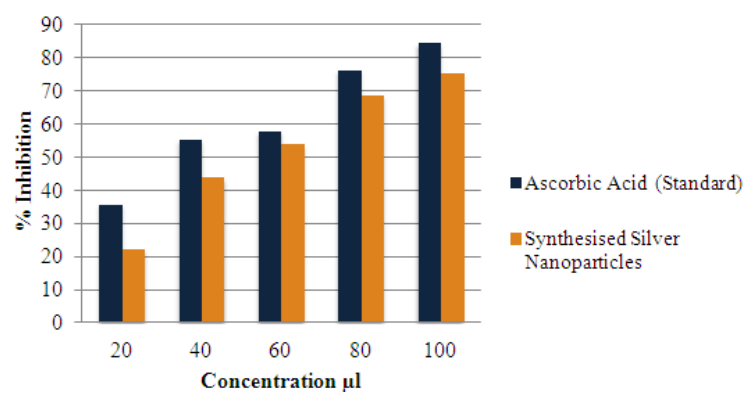

FIGURE 6 Antioxidant Property Of Synthesised Silver Nanoparticles Using Bauhinia tomentosa

\section{Conclusion:}

The green synthesis of metal nanoparticles has wide application in medicinal field due its biocompatibility property and low toxicity. The investigation was deals with Bauhinia tomentosa mediated synthesis of silver nanoparticles was confirmed by color change into reddish brown and further confirmed with UV- Visible spectra the peak obtained at $440.0 \mathrm{~nm}$ and functional groups presence was confirmed with FT-IR spectra. The antibacterial activity was performed against Escherichia coli, Salmonella typhi and Staphylococcus aureus. The antioxidant property was done using ascorbic acid as standard, which showed the gradual increase of antioxidant activity with concentration increases. Thus, the investigation eye opens young scientists to shine their research into the green synthesis of metal nanoparticles which can be utilized for pharmaceutical purpose in future.

\section{Acknowledgement}

I record my sincere thanks to the institute which provides necessary facilities to complete the research work.

\section{References:}

[1] Shakeel Ahmeed, Mudasir Ahmad, Babu Ll Swami, Saiqa Ikram (2016) A Review on Plants Extract Mediated Synthesis of Silver Nanoparticles for Antimicrobial applications: A Green Expertise. Journal of Advanced Resreach 7, 117- 28.

[2] Mahendra Rai, Alka Yadav (2013) Plants As Potential Synthesiser of Precious Metal Nanoparticles: Progress And Prospects IET Nanotechnology. 117- 124.

[3] Balamurughan M G, Mohanraj S, Kodhaiyoloo S, Pugalenthi V (2014) Ocimum sanctum leaf extract mediated green synthesis of iron oxide nanoparticles: spectroscopic and microscopic studies. Journal of Chemical and Pharmaceutical Sciences, 201- 204.

[4] Gopukumar S T, Sana Fathima T K, Princy Alexander, Vinotha Alex, Praseetha P K (2016) Evaluation of Antioxidant Propeties of Silver Nanoparticle Embedded Medicinal Patch, Nanomedicine and Nanotechnology Open acess.

[5] Madhu Kumari(2012) Evaluation of Ethanolic Extracts of In Vitro grown Bauhinia Variegatal and For Antibacterial Activities. Int J Pharm Bio Sci: 43- 50.

[6] Gopalakrishan S, Vadivel E (2011) Antibacterial And Antifungal activity of the Bark of Bauhinia tomentosa. An International Journal of Pharmaceutical Sciences: Vol 2, Issue3. 